\begin{tabular}{|c|c|c|}
\hline & $\begin{array}{c}\text { Gazi University } \\
\text { Journal of Science }\end{array}$ & 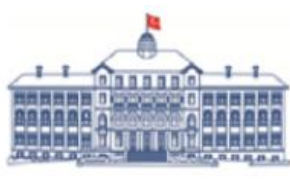 \\
\hline & http://dergipark.gov.tr/gujs & \\
\hline
\end{tabular}

\title{
A Novel Numerical Approach for Solving Convection-Diffusion Problem with Boundary Layer Behavior
}

\author{
Musa CAKIR ${ }^{1}$ (D) , R. Younis MASIHA ${ }^{2}$ (D) Derya ARSLAN $^{3, *}$ \\ ${ }^{1,2}$ Department of Mathematics, Faculty of Science, University of Van Yuzuncu Yil, Van, Turkey \\ ${ }^{3}$ Department of Mathematics, Faculty of Art and Science, University of Bitlis Eren, 13200, Bitlis, Turkey
}

\author{
Highlights \\ - The paper focused on the numerical solution of singularly perturbed boundary value problem. \\ - To obtain the approach of difference scheme, finite difference method is used. \\ - Stability and uniform convergence of presented difference method are investigated. \\ - The examples are solved by using Thomas algorithm.
}

\section{Article Info}

Received: $21 / 03 / 2019$

Accepted: 16/07/2019

\section{Keywords}

Singular perturbation

Finite difference

scheme

Uniform mesh

Uniform convergence

\begin{abstract}
This study deals with a new approach method for solving singularly perturbed boundary value problem of convection-diffusion type. Firstly, bounds on the solution and its derivative of solution to be used later in the article are obtained. This robust method is constructed with fitted difference scheme on a uniform mesh. It is proved that the presented method is first-order convergent with respect to the perturbation parameter $\varepsilon$ in the discrete maximum norm. Two examples are given to illustrate the efficiency of the method. The numerical results are presented in tables and figures.
\end{abstract}

\section{INTRODUCTION}

Here we handle the following convection-diffusion problem with boundary layer behavior:

$\varepsilon u^{\prime \prime}(x)+a(x) u^{\prime}(x)-b(x) u(x)=f(x), 0<x<l$,

$u(0)=A, u(l)=B$,

where $A$ and $B$ are given constants and $0<\varepsilon \ll 1$ is a very small positive parameter. It is supposed that the functions $a(x), b(x)$ and $f(x)$ are continuous differentiable in interval $[0, l]$ and besides, our conditions are

$a(x) \geq \alpha>0$,

$b(x) \geq \beta>0$,

Differential equations with a small parameter $\varepsilon$ multiplying the highest-order derivative terms are called singularly perturbed differential equations. Standard numerical methods for solving singularly perturbed problems are unstable and do not give accurate results due to the perturbation parameter $\varepsilon$. If suitable numerical methods such as finite difference method and finite element method [1-8] for solving these 
problems are developed, then the stable and accurate results are obtained. Therefore, we prefer to apply finite difference method for this problem in this paper.

There are the various approaches to the design and analysis of appropriate numerical methods for singularly perturbed differential equations in [9-17] and the references therein. Singular perturbation problems are located in various fields. For example, chemical-reactor theory, control theory, oceanography, fluid mechanics, quantum mechanics, hydro mechanical problems, meteorology, electrical networks and other physical models [18-25]. There is study of existence and uniqueness of singularly perturbed problems in [26].

The present study is organized as follows: In Section 2, we state some required properties of the exact solution for analysis of numerical method. Then, the finite difference discretization is given in Section 3 . In Section 4, we investigate the error evaluation on uniform mesh for the approximate solution of the problem (1)-(2). Furthermore, numerical results supporting the theory are presented. The obtained results are shown via tables and figures.

Throughout the paper, $C, C_{0}, C_{1}$ denote generic positive constants independent of $\varepsilon$ and the mesh parameter. For any continuous function $g(x)$ denote norms which $g_{\infty} \equiv g_{\infty,[0, l]}=\max _{0 \leq x \leq l}|g(x)|$.

\section{SOME PROPERTIES OF CONTINUOUS PROBLEM}

Here we give asymptotic estimates for the solution of continuous problem (1)-(2). These estimates will be used to analysis of the uniform convergence of difference approximations in the next sections.

Lemma 2.1. Let us accept that the function $u(x)$ is the solution of (1)-(2) and $a(x), b(x), f(x) \in C^{1}[0, l]$. Then the equalities

$u_{\infty} \leq C_{0}$,

$C_{0}=|A|+|B|+\alpha^{-1} f_{\infty}$,

and

$\left|u^{\prime}(x)\right| \leq C\left\{1+\frac{1}{\varepsilon} e^{-\frac{\alpha x}{\varepsilon}}\right\}$,

hold.

Proof. We first show the proof of (5). Here we define the maximum principle for the problem (1)-(2).

Let $L$ be the differential operator in (1)-(2) and $\kappa \in C^{2}[0, l]$ if $\kappa(0) \geq 0, \kappa(l) \geq 0$ and $L \kappa \leq 0$, $0<x<l$, then $\kappa(x) \geq 0$ for $0 \leq x \leq l$.

Using the barrier function, we have

$\Psi^{ \pm}(x)= \pm u(x)+|A|+|B|+\alpha^{-1}(l-x) f_{\infty}$.

By applying the maximum principle to the function above, we find

$$
\begin{aligned}
& \Psi^{ \pm}(0)= \pm A+|A|+|B|+\alpha^{-1}(l-x) f_{\infty} \geq 0, \\
& \Psi^{ \pm}(0)= \pm B+|B|+|A|+|A| \geq 0,
\end{aligned}
$$

and 


$$
\begin{aligned}
& L \Psi(x)= \pm f(x)-(|A|+|B|) b(x)+\alpha^{-1}[a(x)-b(x)](l-x) f_{\infty} \\
& \leq-(|A|+|B|) b(x) \pm|f(x)|-f_{\infty} \leq 0
\end{aligned}
$$

According to maximum principle, we get

$\Psi(x) \geq 0$.

From this inequality, we obtain

$\pm u(x)+|A|+|B|+\frac{(l-x) f_{\infty}}{\alpha} \geq 0$,

$|u(x)|=|A|+|B|+\frac{1}{\alpha}(l-x) f_{\infty}$,

which gives the proof of (5).

We now show the proof of (4). We rewrite the problem (1)-(2) as

$\varepsilon u^{\prime \prime}(x)+a(x) u^{\prime}(x)=F(x)$,

where

$F(x)=f(x)+b(x) u(x)$.

From the relation (7), we have

$u^{\prime}(x)=u^{\prime}(0) e^{-\frac{1}{\varepsilon} \int_{0}^{x} a(\tau) d \tau}+\frac{1}{\varepsilon} \int_{0}^{x} F(\xi) e^{-\frac{1}{\varepsilon} \int_{\xi}^{x} a(\tau) d \tau} d \xi$.

We need an estimate for $u^{\prime}(0)$ in (8). Integrating this equality over $(0, l)$, we have

$B-A=u^{\prime}(0) \int_{0}^{l} e^{-\frac{1}{\varepsilon} \int_{0}^{x} a(\tau) d \tau} d x+\frac{1}{\varepsilon} \int_{0}^{l} \int_{0}^{x} F(\xi) e^{-\frac{1}{\varepsilon} \int_{\xi}^{x} a(\tau) d \tau} d \xi d x$.

From this equality we obtain

$u^{\prime}(0)=\frac{B-A-\frac{1}{\varepsilon} \int_{0}^{l} \int_{0}^{x} F(\xi) e^{-\frac{1}{\varepsilon} \int_{\xi}^{x} a(\tau) d \tau} d \xi d x}{\int_{0}^{l} e^{--\frac{1}{\varepsilon} \int_{0}^{x} a(\tau) d \tau} d x}$.

We evaluate integral in denominator of (9) as

$\int_{0}^{l} e^{-\frac{1}{\varepsilon} \int_{0}^{x} a(\tau) d \tau} d x \geq \int_{0}^{l} e^{-\frac{1}{\varepsilon} \int_{0}^{x} a^{*} d \tau} d x=\int_{0}^{l} e^{-\frac{a^{*} x}{\varepsilon}} d x=\frac{\varepsilon\left(1-e^{-\frac{a^{*} l}{\varepsilon}}\right)}{a^{*}}=\gamma_{0} \varepsilon$,

where $a^{*}=\max _{[0, l]}\{a(x)\}$.

Applying the mean value theorem to integral in (9), we find

$\left|\frac{1}{\varepsilon} \int_{0}^{l}\left[\int_{0}^{x} F(\xi) \exp \left(-\frac{1}{\varepsilon} \int_{\xi}^{x} a(\tau) d \tau\right) d \xi\right] d x\right| \leq \frac{1}{\varepsilon} \int_{0}^{l}\left[\int_{0}^{x}|F(\xi)| \exp \left(-\frac{1}{\varepsilon} \int_{\xi}^{x} a(\tau) d \tau\right) d \xi\right] d x$ 
$\leq \frac{F_{\infty}}{\varepsilon} \int_{0}^{l} \int_{0}^{x} e^{-\frac{\alpha(x-\xi)}{\varepsilon}} d \xi d x=\alpha^{-1} F_{\infty} \int_{0}^{l}\left[1-e^{-\frac{\alpha x}{\varepsilon}}\right] d x \leq \frac{F_{\infty} l}{\alpha} \leq C_{1}$.

By writing the evaluations (10) and (11) in (9), we get

$\left|u^{\prime}(0)\right| \leq \frac{|A|+|B|+C_{1}}{\gamma \varepsilon}=\frac{C}{\varepsilon}$.

Using the estimate (12) in (8), we have

$\left|u^{\prime}(x)\right| \leq \frac{C}{\varepsilon} e^{-\frac{\alpha x}{\varepsilon}}+\frac{F_{\infty}}{\alpha}\left(1-e^{-\frac{\alpha x}{\varepsilon}}\right) \leq \frac{C}{\varepsilon} e^{-\frac{\alpha x}{\varepsilon}}+\frac{F_{\infty}}{\alpha}$,

which gives proof of (6). So, we have completed the proof of Lemma 2.1.

\section{CONSTRUCTION OF DIFFERENCE SCHEMES}

Let $\omega_{N}$ be a uniform mesh on $[0, l]$ as follows:

$\omega_{N}=\left\{x_{i},=i h, i=1,2, \ldots, N-1 ; \quad h=\frac{l}{N}\right\}$,

$\varpi_{N}=\omega_{N} \cup\{x=0, x=l\}$.

Before describing our numerical method, we introduce some notation for the mesh functions. For any mesh function $g(x)$ defined on $\bar{\omega}_{N}$ we use

$$
\begin{aligned}
& g_{i}=g\left(x_{i}\right), \quad g_{\bar{x}, i}=\frac{g_{i}-g_{i-1}}{h}, \quad g_{x, i}=\frac{g_{i+1}-g_{i}}{h}, \quad g_{x, i}=\frac{g_{i+1}-g_{i-1}}{2 h}, \quad u_{\bar{x} x, i}=\frac{g_{i+1}-2 g_{i}+g_{i-1}}{h^{2}}, \\
& g_{1} \equiv g_{1, \omega_{N}}=h \sum_{i=1}^{n-1}\left|g_{i}\right| .
\end{aligned}
$$

Here we construct difference approximate on a uniform mesh for the problem (1)-(2). To obtain the approach of difference scheme, we will integrate equation (1) over $\left(x_{i-1}, x_{i+1}\right)$.

$$
h^{-1} \int_{x_{i-1}}^{x_{i+1}} \operatorname{Lu} \varphi_{i}(x) d x=h^{-1} \int_{x_{i-1}}^{x_{i+1}} f(x) \varphi_{i}(x) d x,
$$

where the basis functions $\varphi_{i}(x)$ are as follows:

$$
\varphi_{i}(x)= \begin{cases}\varphi_{i}^{(1)}(x)=\frac{-1+\exp \left(-\frac{a_{i}\left(x-x_{i-1}\right)}{\varepsilon}\right)}{-1+\exp \left(-\frac{a_{i} h}{\varepsilon}\right)}, & x \in\left(x_{i-1}, x_{i}\right), \\ \varphi_{i}^{(2)}(x)=\frac{1-\exp \left(-\frac{a_{i}\left(x_{i+1}-x\right)}{\varepsilon}\right)}{1-\exp \left(-\frac{a_{i} h}{\varepsilon}\right)}, & x \in\left(x_{i}, x_{i+1}\right), \\ 0, & x \notin\left(x_{i-1}, x_{i+1}\right) .\end{cases}
$$

The functions $\varphi_{i}^{(1)}(x)$ and $\varphi_{i}^{(2)}(x)$ are the solution of the following problems, respectively: 


$$
\begin{aligned}
& \varepsilon \varphi_{i}^{\prime \prime}-a_{i} \varphi_{i}^{\prime}=0, \quad x \in\left(x_{i-1}, x_{i}\right), \\
& \varphi_{i}\left(x_{i-1}\right)=0, \varphi_{i}\left(x_{i}\right)=1, \\
& \varepsilon \varphi_{i}^{\prime \prime}-a_{i} \varphi_{i}^{\prime}=0, \quad x \in\left(x_{i}, x_{i+1}\right), \\
& \varphi_{i}\left(x_{i}\right)=1, \varphi_{i}\left(x_{i+1}\right)=0 .
\end{aligned}
$$

We can continue from (13) as

$$
\begin{aligned}
& \frac{1}{h} \int_{x_{i-1}}^{x_{i+1}}\left[\varepsilon u^{\prime \prime}(x)+a(x) u^{\prime}(x)-b(x) u(x)\right] \varphi_{i}(x) d x=\frac{1}{h} \int_{x_{i-1}}^{x_{i+1}} f(x) \varphi_{i}(x) d x, \\
& \frac{1}{h} \int_{x_{i-1}}^{x_{i+1}}-\varepsilon u^{\prime}(x) \varphi_{i}^{\prime}(x) d x+\frac{1}{h} \int_{x_{i-1}}^{x_{i+1}} a_{i} u^{\prime}(x) \varphi_{i}(x) d x-\frac{1}{h} \int_{x_{i-1}}^{x_{i+1}} b(x) u(x) \varphi_{i}(x) d x=f_{i}+\bar{R}_{i}, \\
& \frac{1}{h} \int_{x_{i-1}}^{x_{i+1}}-\varepsilon u^{\prime}(x) \varphi_{i}^{\prime}(x) d x+\frac{1}{h} \int_{x_{i-1}}^{x_{i+1}} a_{i} u^{\prime}(x) \varphi_{i}(x) d x-b_{i} u_{i}=f_{i}+R_{i},
\end{aligned}
$$

where we can express the reminder term $R_{i}$ as

$$
R_{i}=\bar{R}_{i}+R_{b, i}=R_{a, i}+R_{f, i}+R_{b, i},
$$

with

$$
\begin{aligned}
& R_{a, i}=\frac{1}{h} \int_{x_{i-1}}^{x_{i+1}} u^{\prime}(x)\left[a\left(x_{i}\right)-a(x)\right] \varphi_{i}(x) d x, \\
& R_{b, i}=\frac{1}{h} \int_{x_{i-1}}^{x_{i+1}}\left[b(x) u(x)-b\left(x_{i}\right) u\left(x_{i}\right)\right] \varphi_{i}(x) d x, \\
& R_{f, i}=\frac{1}{h} \int_{x_{i-1}}^{x_{i}}\left[f(x)-f\left(x_{i}\right)\right] \varphi_{i}(x) d x .
\end{aligned}
$$

Using the quadrature rules in (2.1) and (2.2) from [1], we obtain the following precise relation:

$$
\begin{aligned}
& h^{-1} \int_{x_{i-1}}^{x_{i}}\left[\varepsilon u^{\prime} \varphi_{i}^{(1)^{\prime}}(x)+a_{i} u^{\prime}(x) \varphi_{i}^{(1)}(x)\right] d x+h^{-1} \int_{x_{i}}^{x_{i+1}}\left[\varepsilon u^{\prime} \varphi_{i}^{(2)}(x)+a_{i} u^{\prime}(x) \varphi_{i}^{(2)}(x)\right] d x \\
& =-\varepsilon h^{-1} u_{\bar{x}, i}+a_{i} u_{\bar{x}, i} h^{-1} \int_{x_{i-1}}^{x_{i}} \varphi_{i}^{(1)}(x) d x-\varepsilon h^{-1} u_{\bar{x}, i}+a_{i} u_{x, i} h^{-1} \int_{x_{i}}^{x_{i+1}} \varphi_{i}^{(2)}(x) d x, \\
& =\varepsilon \frac{u_{x, i}-u_{\bar{x}, i}}{h}+a_{i} u_{\bar{x} \bar{i}} \sigma_{i}^{(1)}+a_{i} u_{x} \sigma_{i}^{(2)}=\varepsilon u_{\bar{x} x, i}+a_{i} u_{\bar{x}, i} \sigma^{(1)}+a_{i} u_{x, i} \sigma^{(2)},
\end{aligned}
$$

where

$$
\sigma_{i}^{(1)}=h^{-1} \int_{x_{i-1}}^{x_{i}} \varphi_{i}^{(1)}(x) d x=h^{-1} \int_{x_{i-1}}^{x_{i}} \frac{-1+\exp \left(-\frac{a_{i}\left(x-x_{i-1}\right)}{\varepsilon}\right)}{-1+\exp \left(-\frac{a_{i} h}{\varepsilon}\right)} d x=\frac{\varepsilon}{a_{i} h}+\frac{1}{1-e^{a_{i} h / \varepsilon}},
$$


$\sigma_{i}^{(2)}=h^{-1} \int_{x_{i}}^{x_{i+1}} \varphi_{i}^{(2)}(x) d x=h^{-1} \int_{x_{i}}^{x_{i+1}} \frac{1-\exp \left(-\frac{a_{i}\left(x_{i+1}-x\right)}{\varepsilon}\right)}{1-\exp \left(-\frac{a_{i} h}{\varepsilon}\right)}=\frac{1}{e^{a_{i} h / \varepsilon}-1}-\frac{\varepsilon}{a_{i} h}$.

Using the relations

$u_{x, i}-u_{\bar{x}, i}=\frac{h}{2} u_{\bar{x}_{x, i}}$,

and

$u_{x, i}-u_{x, i}=\frac{h}{2} u_{x, i}$,

in (19), we obtain

$\varepsilon u_{\bar{x} x, i}+a_{i} \sigma_{i}^{(1)} u_{0, i},-a_{i} \sigma_{i}^{(1)} \frac{h}{2} u_{\bar{x}_{x, i}}-a_{i} \sigma_{i}^{(2)} u_{x, i}+a_{i} \sigma_{i}^{(2)} \frac{h}{2} u_{\bar{x}_{x, i}}$
$=\varepsilon\left\{1+\frac{a_{i} h}{\varepsilon}\left(\sigma_{i}^{(2)}-\sigma_{i}^{(1)}\right)\right\} u_{\bar{x}_{x, i}}+a_{i}\left(\sigma_{i}^{(1)}+\sigma_{i}^{(2)}\right) u_{x, i}=\varepsilon \theta_{i} u_{\bar{x} x, i}+a_{i} u_{x, i}$,

where

$\theta_{i}=\varepsilon\left\{1+\frac{a_{i} h}{\varepsilon}\left(\sigma_{i}^{(2)}-\sigma_{i}^{(1)}\right)\right\}=\gamma_{i} \operatorname{coth}\left(\gamma_{i}\right), \gamma_{i}=\frac{a_{i} h}{2 \varepsilon}$,

$\sigma_{i}^{(1)}+\sigma_{i}^{(2)}=h^{-1} \int_{x_{i-1}}^{x_{i+1}} \varphi_{i}(x) d x=1$.

Then, by using the relation (20) in (14), we get

$l y_{i} \equiv \varepsilon \theta_{i} y_{\bar{x} x, i}+a_{i} y_{\substack{o, i \\ x, i}}-b_{i} y_{i}=f_{i}+R_{i}, \quad 1 \leq i \leq N-1$.

Neglecting $R_{i}$ in the relation (22), we propose the following finite difference approximate for the problem (1)-(2):

$$
\begin{aligned}
& l y_{i} \equiv \varepsilon \theta_{i} y_{\overline{x x}_{i}}+a_{i} y_{\substack{x, i \\
x, i}}-b_{i} y_{i}=f_{i}, \\
& y_{0}=A, y_{N}=B,
\end{aligned}
$$

where $\theta_{i}$ is defined by (21).

\section{STABILITY AND $\varepsilon-$ UNIFORM CONVERGENCE}

Here we will give stability and uniform convergence of presented difference method.

Lemma 4.1. If we accept the smoothness assumptions of Lemma 2.1, then the truncation function $R$ satisfies the following inequality.

$\|R\|_{\infty, \omega_{N}} \leq C h$.

Proof. We first show that

$\left\|R_{f}\right\|_{\infty, \omega_{N}} \leq C h$. 
Applying the mean value theorem to function in (18), we get

$\left|\frac{f(x)-f\left(x_{i}\right)}{x-x_{i}}\right|=\left|f^{\prime}(\xi)\right| \leq \max \left|f^{\prime}(x)\right|\left|x-x_{i}\right| \leq C, \quad \xi \in\left(x_{i}, x\right)$.

Hence, we obtain

$\left\|R_{f}\right\|_{\infty, \omega_{N}} \leq C h$.

In second case let us indicate that

$\left\|R_{a}\right\|_{\infty, \omega_{N}} \leq C h$.

By applying the mean value theorem to the function in (16), we have

$\left|\frac{a(x)-a\left(x_{i}\right)}{x-x_{i}}\right|=\left|a^{\prime}(\xi)\right| \leq \max \left|a^{\prime}(\xi)\right|\left|x-x_{i}\right| \leq C_{0} h, \xi \in\left(x_{i}, x\right)$.

From this, we obtain

$$
\begin{aligned}
& \left\|R_{a}\right\|_{l_{1}\left(\omega_{N}\right)} \leq C_{0} h \sum_{i=1}^{N-1} \int_{x_{i-1}^{x_{i+1}}} C_{1}\left(1+\frac{1}{\varepsilon} e^{\frac{-\propto x}{\varepsilon}}\right) d \leq C_{0} C_{1} h\left(2 l+\sum_{i=1}^{N-1} \int_{x_{i-1}}^{x_{i+1}} \frac{1}{\varepsilon} e^{\frac{-\propto x}{\varepsilon}} d x\right) \\
& \leq C_{0} C_{1} h\left(2 l+\frac{2}{\varepsilon} \int_{0}^{l} e^{\frac{-\propto x}{\varepsilon}}\right) d x \leq C h .
\end{aligned}
$$

In the same way, we can demonstrate the following inequality as similar to the inequality (27).

$\left\|R_{b}\right\|_{l_{1}\left(\omega_{N}\right)} \leq C h$.

Applying the mean value theorem to the function in the relation (17), we take

$$
\begin{aligned}
& \left|\frac{b(x) u(x)-b\left(x_{i}\right) u\left(x_{i}\right)}{x-x_{i}}\right|=\left|(b(\xi) u(\xi))^{\prime}\right| \leq \max \left|u(x) b^{\prime}(x)+u^{\prime}(x) b(x)\right|\left|x-x_{i}\right| \\
& \leq\left(\max \left|u(x) b^{\prime}(x)\right|+\max \left|u^{\prime}(x) b(x)\right|\right)\left|x-x_{i}\right| \leq c_{1} h, x_{i}<\xi<x .
\end{aligned}
$$

From this, we get

$$
\left\|R_{b}\right\|_{l_{1}\left(\omega_{N}\right)} \leq c_{1} h \sum_{i=1}^{N-1} \int_{x_{i-1}}^{x_{i+1}} c_{1}\left(1+\frac{1}{\varepsilon} \exp \left(-\frac{\alpha x}{\varepsilon}\right)\right) d x \leq c_{1} h,
$$

which leads to (28).

Hence, from the inequalities (26), (27) and (28) we have (25).

Lemma 4.2. Error function $z_{i}, 0 \leq i \leq N$ satisfies the following inequality:

$$
\left|z_{i}\right| \leq C R_{l_{1}\left(\varpi_{N}\right)}=C h \sum_{i=1}^{n-1}\left|R_{i}\right| .
$$

Proof. According to maximum principle, we have

$\left|z_{i}\right| \leq \eta_{i}, \quad i=1,2, \cdots, N-1$,

where the function $\eta_{i}$ is the solution of the following difference problem. 
$\varepsilon \theta_{i} \eta_{x x, i}+a_{i} \eta_{x, i}=-\left|R_{i}\right|$,

$\eta_{0}=0, \eta_{N}=0$.

Let $\eta_{x, i}=V_{i}$. Then we rewrite the relation (30) as

$\varepsilon \theta_{i} V_{\bar{x}, i}+a_{i} \frac{V_{i}+V_{i-1}}{2}=-\left|R_{i}\right|$.

Solving the first order difference with respect to $V_{i}$ for $1 \leq i \leq N-1$, we obtain

$V_{i}=V_{0} \prod_{k=1}^{i}\left(\frac{1-\frac{h a_{k}}{2 \varepsilon \theta_{k}}}{1+\frac{h a_{k}}{2 \varepsilon \theta_{k}}}\right)-h \sum_{l=1}^{i} \frac{\left|R_{i}\right|}{\varepsilon \theta_{l}+\frac{h a_{i}}{2}}$.

Using the inequality $1-x \leq e^{-x}$ and $\frac{a_{k} \rho}{2 \theta_{k}}=\cot h\left(\frac{a_{k} \rho}{2}\right) \leq 1$ we get

$\left|V_{i}\right|=\left|V_{0}\right| \exp \left(-h \sum_{k=1}^{i} \frac{\frac{a_{k}}{\varepsilon \theta_{k}}}{1+\frac{a_{k}}{2 \varepsilon \theta_{k}}}\right)+\sum_{l=1}^{i} \frac{\left|R_{i}\right|}{\varepsilon \theta_{l}+\frac{h a_{i}}{2}} \times\left(-h \sum_{j=l+1}^{i} \frac{a_{j} / \varepsilon \theta_{j}}{\varepsilon \theta_{l}+\frac{h a_{l}}{2}}\right)$.

For the second term in the relation (33), we found

$\max _{1 \leq l \leq N-1} \frac{1}{\varepsilon \theta_{l}+\frac{h a_{l}}{2}} \exp \left(-h \sum_{j=l+1}^{i} \frac{\frac{a_{j}}{\varepsilon \theta_{j}}}{1+\frac{h a_{j}}{2 \varepsilon \theta_{k}}}\right) \leq C$.

From this, the second term in the relation (33) is obtained as follows.

$h \sum_{l=1}^{i} \frac{\left|R_{l}\right|}{\varepsilon \theta_{l}+\frac{a_{l} h}{2}} \exp \left(-h \sum_{j=l+1}^{i} \frac{a_{j} / \varepsilon \theta_{j}}{\varepsilon \theta_{l}+h a_{l} / 2}\right) \leq C h \sum_{l=1}^{i}\left|R_{l}\right|$,

and

$$
V_{0}=\frac{h \sum_{i=0}^{N-1}\left\{h \sum_{l=1}^{i} \frac{\left|R_{l}\right|}{\varepsilon \theta_{l}+\frac{a_{l} h}{2}} \prod_{j=l+1}^{i}\left(\frac{1-a_{j} h / 2 \varepsilon \theta_{j}}{1+a_{j} h / 2 \varepsilon \theta_{j}}\right)\right\}}{h \sum_{i=0}^{N-1}\left\{\prod_{k=1}^{i}\left(\frac{h a_{k} / \varepsilon \theta_{k}}{1+h a_{k} / 2 \varepsilon \theta_{k}}\right)\right\}} .
$$

By setting the relation (34) in (32), we take 
$\left|V_{0} \prod_{k=1}^{i}\left(\frac{1-\frac{a_{k} h}{2 \varepsilon \theta_{k}}}{1+\frac{a_{k} h}{2 \varepsilon \theta_{k}}}\right)\right| \leq C h \sum_{i=1}^{n-1}\left|R_{i}\right|$.

By using the relation (33) and (35) in (32) we obtain

$\left|v_{i}\right| \leq C h \sum_{i=1}^{n-1}\left|R_{i}\right|$.

So, we have

$\left|z_{i}\right| \leq C h \sum_{i=1}^{n-1}\left|R_{i}\right|$.

The Lemma 4.2 is proven.

We now give the following uniform convergence result.

Theorem 4.3. Suppose that $a, b, f \in C^{1}[0, l]$. Let $u(x)$ be the solution of the problem (1)-(2) and $y$ be the solution of the problem (23)-(24). Uniform error estimate

$\|y-u\|_{C\left(\varpi_{N}\right)} \leq C h$,

holds.

Proof. This follows immediately by combining the two previous lemmas.

\section{NUMERICAL RESULTS}

Here we test two examples for applying of the theoretical results.

Example 5.1. Our first test problem is as follows [27].

$\varepsilon u^{\prime \prime}(x)+\left(1-\frac{x}{2}\right) u^{\prime}(x)-0.5 u(x)=0, \quad x \in(0,1)$,

$u(0)=0, u(1)=1$.

Its exact solution is given by

$u(x)=(2-x)^{-1}-0.5 \exp \left(-\frac{x-\frac{x^{2}}{4}}{\varepsilon}\right)$.

We describe the maximum errors $e_{\varepsilon}^{N}$ and the errors $e^{N}$ as follows:

$e_{\varepsilon}^{N}=\max _{1<i<N}\left|u_{i}^{\varepsilon}-y_{i}^{\varepsilon}\right|, \quad e^{N}=\max _{\varepsilon} e_{\varepsilon}^{N}$.

We also describe the following convergence rates and the computed parameter uniform convergence rates, respectively:

$p_{\varepsilon}^{N}=\frac{\ln \frac{e_{\varepsilon}^{N}}{e_{\varepsilon}^{2 N}}}{\ln 2}, p^{N}=\frac{\ln \frac{e^{N}}{e^{2 N}}}{\ln 2}$. 
Table 1. The calculated maximum errors and the orders of convergence for the numerical solution of problem (37)-(38)

\begin{tabular}{|c|c|c|c|c|c|c|c|}
\hline$\varepsilon / N$ & 8 & 16 & 32 & 64 & 128 & 256 & 512 \\
\hline $10^{-3}$ & 0.0291666 & 0.0151209 & 0.0076884 & 0.0038760 & 0.0019725 & 0.0011393 & 0.0006604 \\
& 0.94 & 0.97 & 0.98 & 0.97 & 0.79 & 0.78 & \\
\hline $10^{-4}$ & 0.0291666 & 0.0151206 & 0.0076884 & 0.0038754 & 0.0019454 & 0.0010393 & 0.0006304 \\
& 0.94 & 0.97 & 0.98 & 0.99 & 0.90 & 0.85 & \\
\hline $10^{-5}$ & 0.0291665 & 0.0151206 & 0.0076884 & 0.0038774 & 0.0019456 & 0.0009754 & 0.0004878 \\
& 0.95 & 0.98 & 0.99 & 0.99 & 0.99 & 0.99 & \\
\hline $10^{-6}$ & 0.0291543 & 0.0151203 & 0.0076884 & 0.0038756 & 0.0019123 & 0.0009798 & 0.0004878 \\
& 95 & 0.98 & 0.99 & 1.00 & 0.97 & 1.00 & \\
\hline $10^{-7}$ & 0.0290543 & 0.0151204 & 0.0076882 & 0.0038742 & 0.0019419 & 0.0009772 & 0.0004878 \\
& 0.95 & 0.98 & 0.99 & 0.99 & 0.99 & 1.00 & \\
\hline $10^{-8}$ & 0.0290342 & 0.0151202 & 0.0076884 & 0.0038750 & 0.0019312 & 0.0009623 & 0.0004878 \\
& 0.94 & 0.98 & 0.99 & 1.00 & 1.00 & 0.98 & \\
\hline $10^{-9}$ & 0.0290540 & 0.0151200 & 0.0076884 & 0.0038751 & 0.0019134 & 0.0009601 & 0.0004878 \\
& 0.94 & 0.98 & 0.99 & 1.01 & 0.99 & 0.98 & \\
\hline $10^{-10}$ & 0.0290342 & 0.0151201 & 0.0076884 & 0.0038705 & 0.0019017 & 0.0009512 & 0.000487 \\
& 0.94 & 0.98 & 0.99 & 1.01 & 0.99 & 0.96 & \\
\hline
\end{tabular}

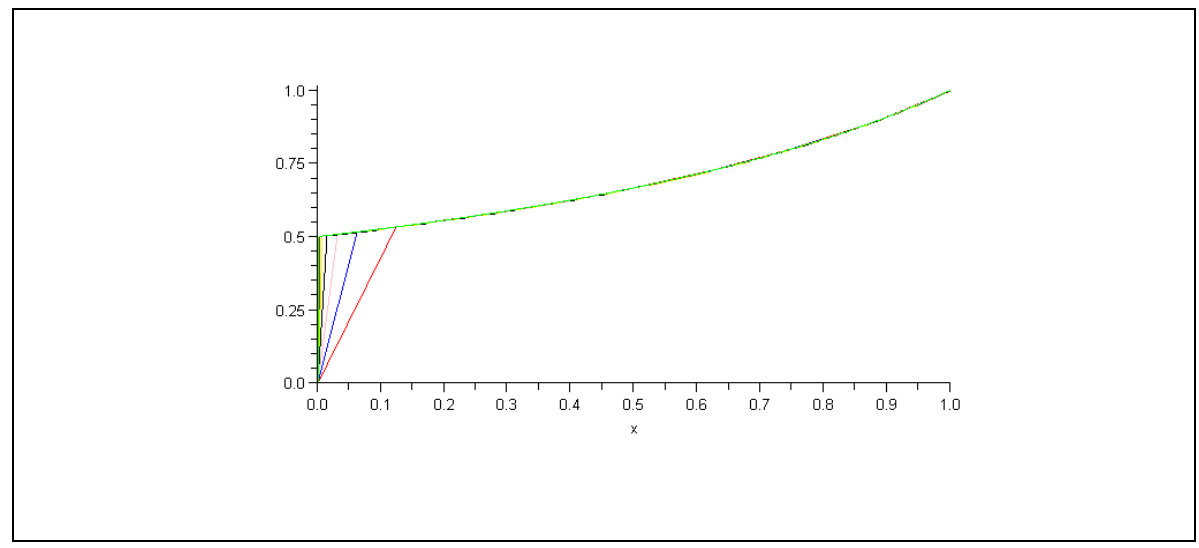

Figure 1. Numerical solution of problem (37)-(38) for different values of $\varepsilon$ and $N$ on the uniform mesh

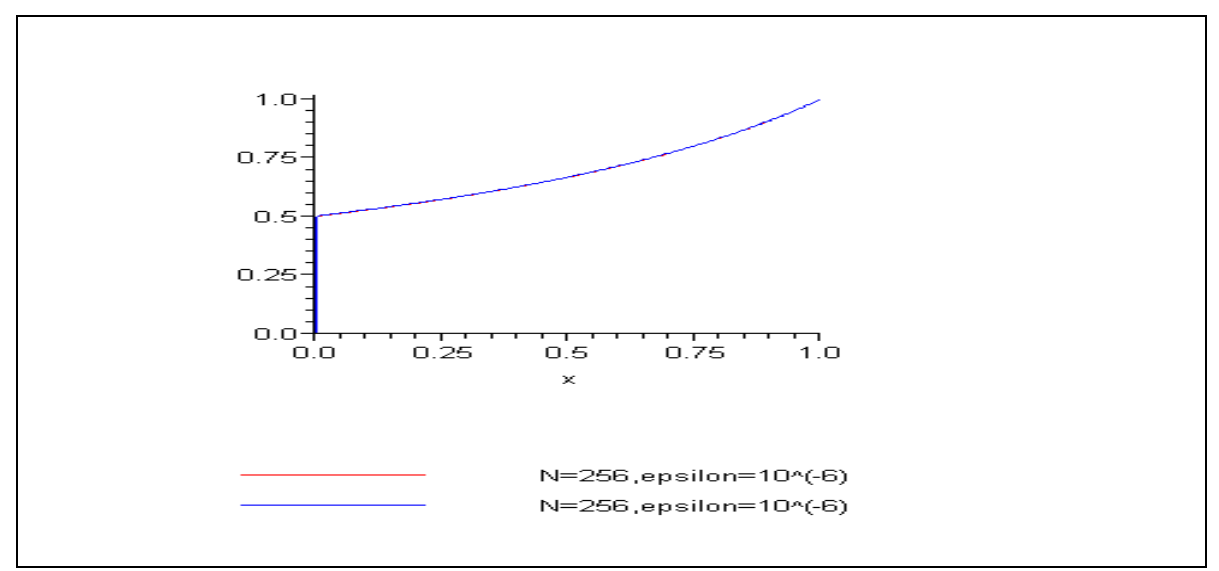

Figure 2. Exact solution and Numerical solution of problem (37)-(38) for varied values of $\varepsilon$ and $N$ on uniform grid 


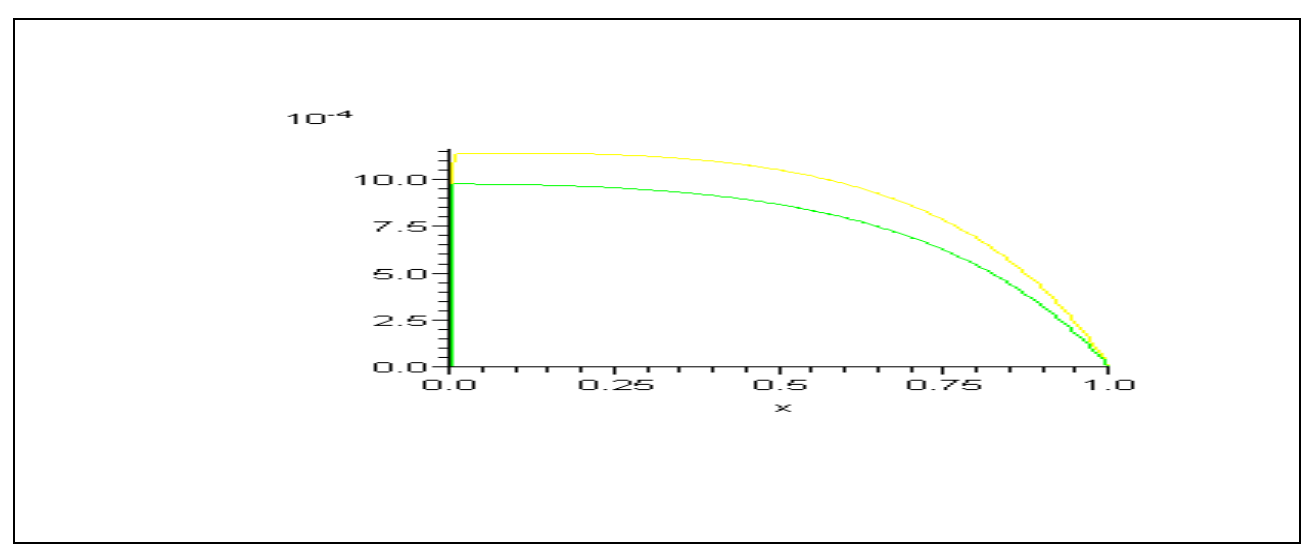

Figure 3. Errors of problem (37)-(38) for values $\varepsilon=10^{-4}, 10^{-6}, 10^{-8}, 10^{-10}$ and $N=256$ on the uniform grid

Example 5.2. We secondly present the following example [27].

$\varepsilon u^{\prime \prime}(x)+u^{\prime}(x)-1-2 x=0, \quad 0<x<1$,

$u(0)=0, u(1)=1$.

Exact solution of example above is as follows.

$u(x)=x^{2}+x-2 x \varepsilon+\frac{(2 \varepsilon-1)\left(1-\exp \left(-\frac{x}{\varepsilon}\right)\right)}{1-\exp \left(-\frac{1}{\varepsilon}\right)}$.

Table 2. The calculated maximum errors and the orders of convergence for the numerical solution of problem (39)-(40)

\begin{tabular}{|c|c|c|c|c|c|c|c|}
\hline$\varepsilon / N$ & 8 & 16 & 32 & 64 & 128 & 256 & 512 \\
\hline $10^{-4}$ & 0.1092000 & 0.0584062 & 0.0300796 & 0.0151839 & 0.0075530 & 0.0036917 & 0.0017497 \\
& 0.90 & 0.95 & 0.98 & 1.00 & 1.03 & 1.07 & \\
\hline $10^{-5}$ & 0.1093575 & 0.0585750 & 0.0302541 & 0.0153611 & 0.0077316 & 0.0038710 & 0.0019293 \\
& 0.90 & 0.95 & 0.97 & 099 & 099 & 1.00 & \\
\hline $10^{-6}$ & 0.1093732 & 0.0585918 & 0.0302715 & 0.0153788 & 0.0077494 & 0.0038889 & 0.0019473 \\
& 0.90 & 0.95 & 0.97 & 0.98 & 0.99 & 0.99 & \\
\hline $10^{-7}$ & 0.1093748 & 0.0585935 & 0.0302732 & 0.0153806 & 0.0077512 & 0.0038907 & 0.0019491 \\
& 090 & 0.95 & 0.97 & 0.98 & 0.99 & 0.99 & \\
\hline $10^{-8}$ & 0.1093749 & 0.0585937 & 0.0302734 & 0.0153808 & 0.0077513 & 0.0038909 & 0.0019499 \\
& 0.90 & 0.95 & 0.98 & 0.99 & 0.99 & 1.00 & \\
\hline $10^{-9}$ & 0.1093749 & 0.0585937 & 0.0302734 & 0.0153808 & 0.0077514 & 0.0038909 & 0.0019493 \\
& 0.90 & 0.95 & 0.98 & 0.99 & 0.99 & 1.00 & \\
\hline $10^{-10}$ & 0.1093749 & 0.0585937 & 0.0302734 & 0.0153808 & 0.0077514 & 0.0038909 & 0.0019491 \\
& 0.89 & 0.95 & 0.98 & 0.99 & 0.99 & 1.00 & \\
\hline
\end{tabular}




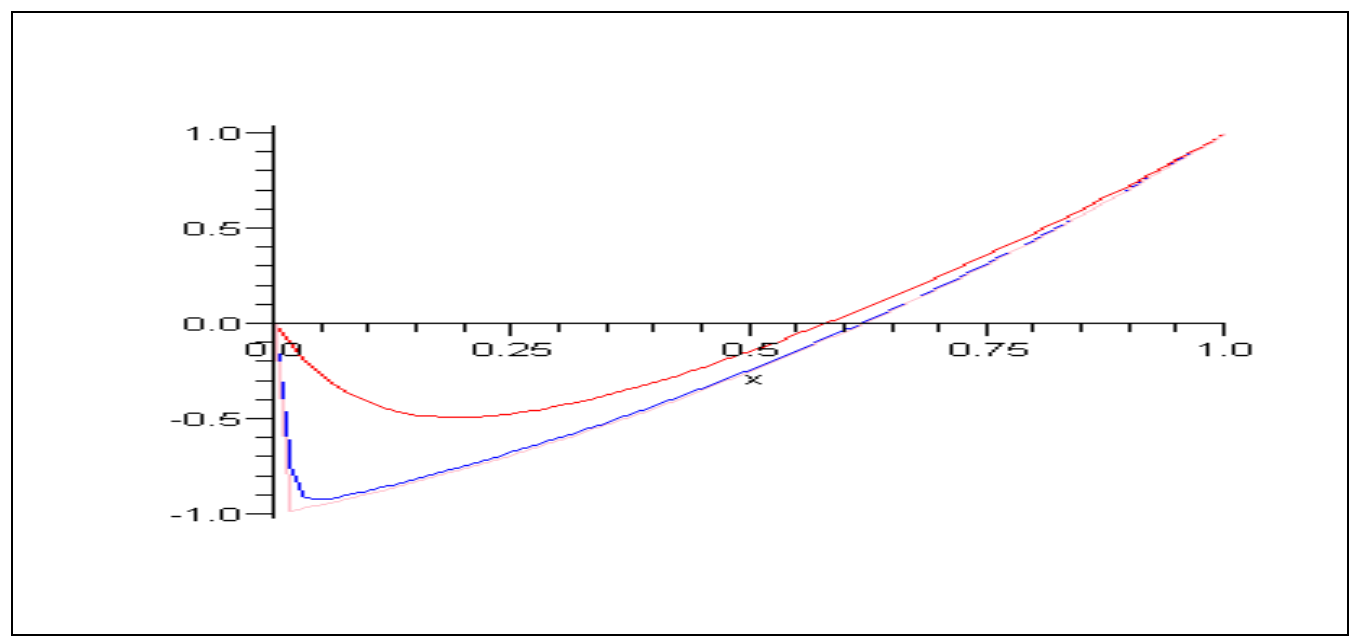

Figure 4. Numerical solution of problem (37)-(38) for values $\varepsilon=10^{-1}, 10^{-5}, 10^{-9}$ and $N=64$ on the uniform mesh

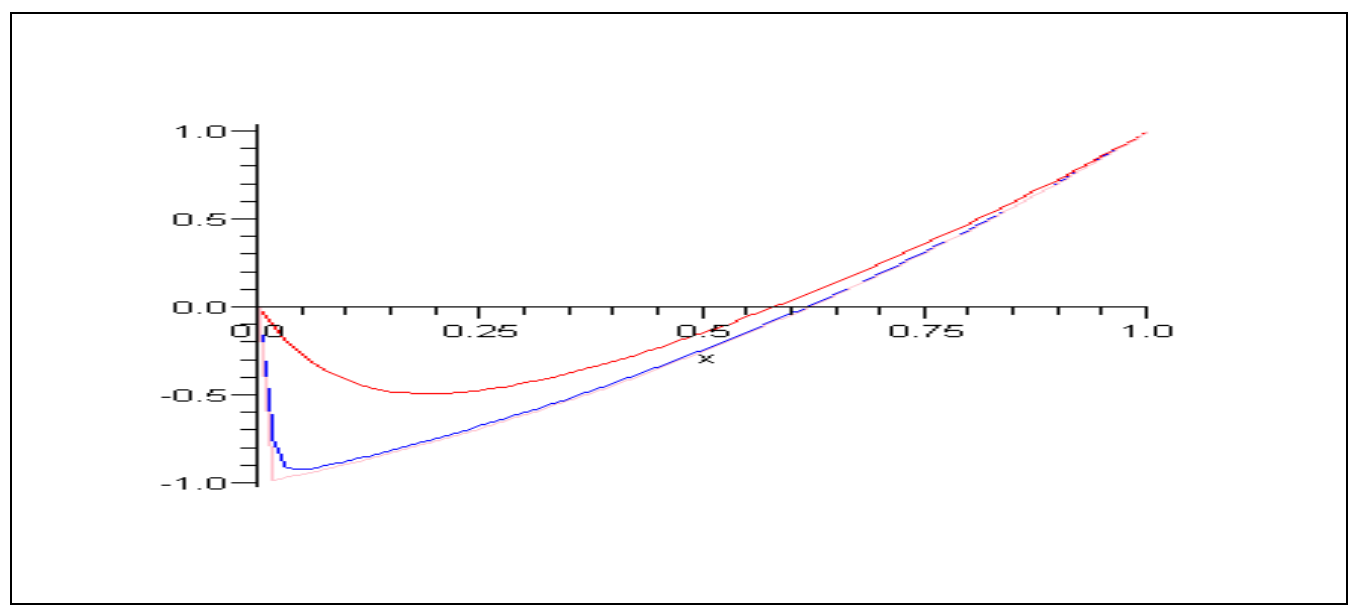

Figure 5. Exact solution and Numerical solution of problem (39)-(40) for values of $\varepsilon=10^{-1}, 10^{-5}, 10^{-9}$ and $N=64$ on the uniform mesh

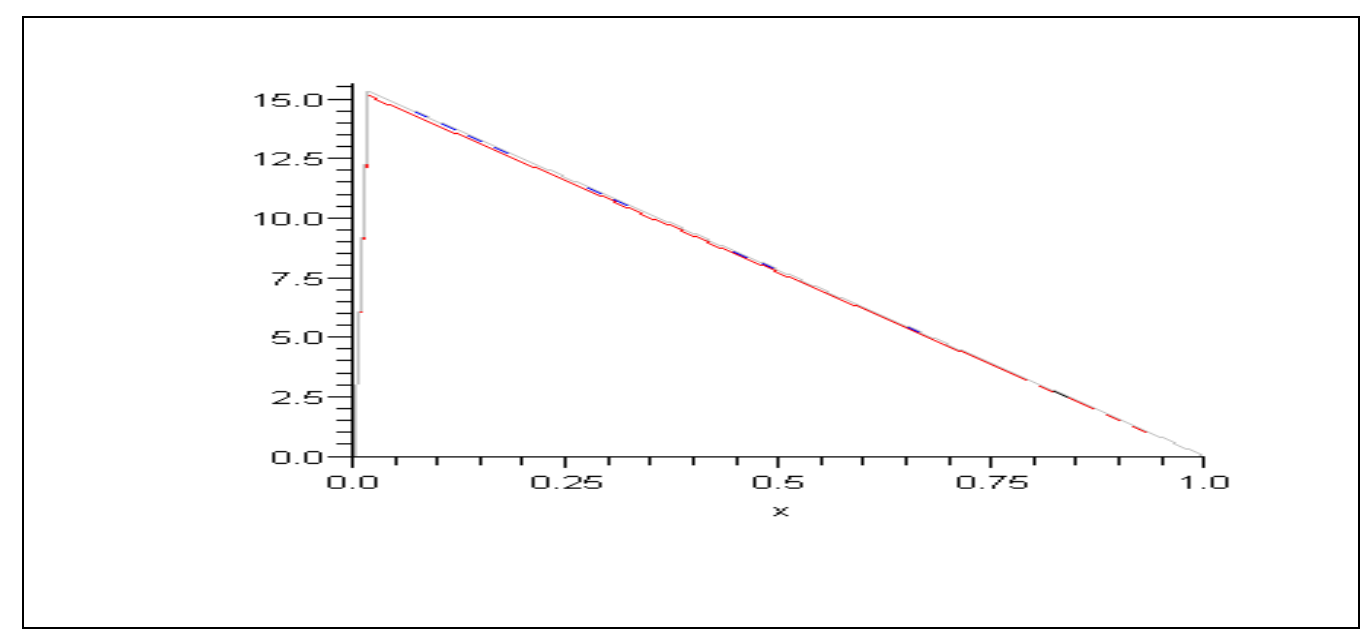

Figure 6. Errors of problem (39)-(40) for values of $\varepsilon=10^{-4}, 10^{-6}, 10^{-8}, 10^{-10}$ and $N=64$ on the uniform mesh 
According to above tables and figures, the curves of exact and approximate solutions are compared in Figures 2 and 5. In Figures 1 and 4, as $\varepsilon$ values decrease, the curves go towards the coordinate axes around $\mathrm{x}=0$. In Figure 3 and 6, the errors in the boundary layer region are maximum for different values $\varepsilon$. The values of $\varepsilon$ and $N$ for which we solve the test problems are $\varepsilon=2^{-k}, k=4, . ., 10 ; N=2^{k}, k=3, . ., 9$. The resulting error $e^{N}$ and the uniform convergence rates $p^{N}$ are given in Tables 1 and 2.

\section{CONCLUSION}

In this study, we have offered an effective finite difference method for solving second-order linear singularly perturbed boundary value problem. It is given that this method displays uniform convergence with respect to the perturbation parameter $\varepsilon$. We apply two examples to show the advantages and effectiveness of this novel approach. The algorithm, figures and tables are generated in Maple. In table and graphics, when $N$ takes increasing values, it is seen that the convergence rate of the smooth convergence speed $p^{N}$ is first order. The curves of exact solutions and approximate solutions are almost identical as shown in Figures 2 and 5. In Figures 1 and 4, as $\varepsilon$ values decrease, the graph approaches more towards the coordinate axes in the boundary layer region around $x=0$. In Figure 3 and 6 , the errors in these regions are maximum because of the irregularity caused by the sudden and rapid change of solution in the boundary layer region around $x=0$ for different values $\varepsilon$. As a result, the proposed scheme has worked very well as numerical results show. This method can also be used for solving other more different problems.

\section{ACKNOWLEDGEMENTS}

The author would like to thank the editor and anonymous referees for their valuable comments and suggestions.

\section{CONFLICTS OF INTEREST}

No conflict of interest was declared by the authors.

\section{REFERENCES}

[1] Amiraliyev, G. M., Mamedov, Y. D., "Difference schemes on the uniform mesh for singularly perturbed pseudo-parabolic equations", Turkish Journal of Mathematics, 19: 207-222, (1995).

[2] Cibık, A., "The effect of a sparse grad-div stabilization on control of stationary Navier-Stokes equations", Journal of Mathematical Analysis and Applications, 437(1): 613-628, (2016).

[3] Amiraliyev, G. M., Çakır, M., "A uniformily convergent difference scheme for singularly perturbed problem with convective term and zeroth order reduced equation", International Journal of Applied Mathematics, 2(12): 1407-1419, (2000).

[4] Cibık, A., Kaya, S., "Finite element analysis of a projection-based stabilization method for the Darcy-Brinkman equations in double-diffusive convection", Applied Numerical Mathematics , 64: 35-49, (2013).

[5] Bakhvalov, N. S., "On optimization of methods for solving boundary-value problems in the presence of a boundary layer", Zhurnal Vychislitel'noi Matematiki i Matematicheskoi Fiziki, 9(4): 841-859, (1969).

[6] Nayfeh, A. H., "Introduction to Perturbation Techniques", Wiley, New York, (1993). 
[7] Bitsadze, A. V., Samarskii, A. A., "On some simpler generalization of linear elliptic boundary value problems", Doklady Akademii Nauk SSSR, 185: 739-740, (1969).

[8] Cakır, M., "Uniform second-order difference method for a singularly perturbed three-point boundary value problem", Hindawi Publishing Corporation Advances in Difference Equations, 13 pages, (2010).

[9] Cakır, M., Amiraliyev, G. M., "Numerical solution of a singularly perturbed three-point boundary value problem", International Journal of Applied Mathematics, 84: 1465- 1481, (2007).

[10] Arslan, D., "Stability and convergence analysis on Shishkin mesh for a nonlinear singularly perturbed problem with three-point boundary condition", Quaestiones Mathematicae, (2019), Doi: 10.2989/16073606.2019.1636894.

[11] Cakır, M., Arslan, D., "A numerical method for nonlinear singularly perturbed multi-point boundary value problem”, Journal of Applied Mathematics and Physics, 4: 1143-1156, (2016).

[12] Cakır, M., Arslan, D., "Finite difference method for nonlocal singularly perturbed problem", International Journal of Modern Research in Engineering and Technology, 1(5): 25-39, (2016).

[13] Cakır, M., Arslan, D., "Numerical solution of the nonlocal singularly perturbed problem", International Journal of Modern Research in Engineering and Technology, 1(5): 13-24, (2016).

[14] Chegis, R., "The Numerical solution of problems with small parameter at higher derivatives and nonlocal conditions", Lietuvos Matematikos Rinkinys (in Russian), 28: 144-152, (1988).

[15] Cimen, E., Amiraliyev, G. M., "A uniform convergent method for singularly perturbed nonlinear differential-difference equation", Journal of Informatics and Mathematical Sciences, 9: 191-199, (2017).

[16] Cimen, E., Cakir, M., "Numerical treatment of nonlocal boundary value problem with layer behaviour", Bulletin of the Belgian Mathematical Society-Simon Stevin, 24, (2017).

[17] Cimen, E., "A priori estimates for solution of singularly perturbed boundary value problem with delay in convection term", Journal of Mathematical Analysis, 8: 202-211, (2017).

[18] Doolan, E. P., Miller, J. J. H., Schilders, W. H. A., "Uniform Numerical Methods for Problem with Initial and Boundary Layers", Boole Press, Dublin, (1980).

[19] Farrel, P. A., Miller, J. J. H., O’Riordan, E., Shishkin, G. I., "A uniformly convergent finite difference scheme for a singularly perturbed semilinear equation", SIAM Journal on Numerical Analysis, 33: 1135-1149, (1996).

[20] Gupta, C. P., Trofimchuk, S. I., "A sharper condition for the solvability of a three-point second order boundary value problem", Journal of Mathematical Analysis and Applications, 205: 586597, (1997).

[21] Miller, J. J. H., O’Riordan, E., Shishkin, G. I., "Fitted numerical methods for singular perturbation problems", World Scientific, Singapore, (1996).

[22] Roos, H. G., Stynes, M., "Tobiska, L., Robust Numerical Methods for Singularly Perturbed Differential Equation: Convection-Diffusion and Flow Problems", Springer-Verlag, Berlin, 604, (2008). 
[23] Cibik, A. B., Y1lmaz, F. N., "Variational multiscale method for the optimal control problems of convection-diffusion-reaction equations", Turkish Journal of Mathematics, 42: 164-180, (2018).

[24] O'Malley, R. E., "Singular Perturbation Methods for Ordinary Differential Equations", Springer Verlag, New York, (1991).

[25] Stynes, M., Roos, H. G., Tobiska, L., "Robust Numerical Methods for Singularly Perturbed Differential Equations", Springer-Verlag, Berlin, (2008).

[26] Jankowski, T., "Existence of solutions of differential equations with nonlinear multipoint boundary conditions", Computers \& Mathematics with Applications, 47: 1095-1103, (2004).

[27] Geng, Z., Tang, Q., "Piecewise shooting reproducing kernel method for linear singularly perturbed boundary value problem", Applied Mathematics Letters, 62: 1-6, (2016). 